\title{
Investigations of the vortex ring state on a helicopter main rotor based on computational methodology using URANS solver
}

\author{
Wienczyslaw Stalewski ${ }^{l}$ and Katarzyna Surmacz ${ }^{l}$ \\ ${ }^{1}$ Lukasiewicz Research Network - Institute of Aviation \\ Al. Krakowska 110/114, 02-256 Warszawa, Poland
}

\begin{abstract}
Computational investigations of the Vortex Ring State (VRS) on a helicopter main rotor have been conducted. The VRS phenomenon is a condition of powered flight that occurs most frequently during the vertical or nearly vertical descent of a rotorcraft. The characteristic feature of the VRS is a torus-shaped vortex around a rotor. The occurrence of this extensive vortex structure is a dangerous phenomenon that usually causes sudden decrease of main-rotor thrust, finally leading to an increase of the rate of descent and vibration level, disturbances of a helicopter balance, deterioration of manoeuvrability and deficit of power. The investigations presented in the paper, have been conducted based on computational methodology developed and implemented by the Authors. The methodology is based on a coupling of several methods of Computational Fluid Dynamics and Flight Dynamic. The approach consists of calculation of unsteady aerodynamic forces acting on the flying rotorcraft by simultaneous solution of the URANS equations, the equations of motion of the helicopter as well as the equations describing fluid-structure-interaction phenomena. Flow effects caused by rotating rotor blades, are modelled using a simplified approach based on the Virtual Blade Model. Using the described methodology, a series of helicopter flight simulations, in a vicinity of the VRS boundaries, have been conducted. Selected results of these simulations have been discussed in the paper.
\end{abstract}

\section{Introduction}

One of the restrictions for use of the helicopter is the Vortex Ring State (VRS) boundary the aerodynamic phenomenon that may occur on both the main or tail rotor. Though both the cases are very dangerous from point of view of helicopter flight safety, the presented study is focused only on the VRS on a helicopter main rotor. In this case, the phenomenon is also known as "settling with power".

The VRS on a helicopter main rotor is a phenomenon that occurs most frequently during a vertical or nearly vertical descent of the rotorcraft. The characteristic feature of the VRS is a toroidal vortex structure developed around the rotor. Visualisations of such structure, taken within both the wind-tunnel tests and flight tests, are presented in Fig. 1.

The reason for the development of the VRS on a main rotor is an interaction between the external flow, resulting from a rotorcraft motion (vertical or nearly vertical descent) and the 
flow induced by the rotor. An occurrence of VRS on a main rotor, may lead to a series of dangerous effects such as increase of helicopter descent rate, increase of vibration level, disturbances of balance, loss of controllability and manoeuvrability or deficit of power.

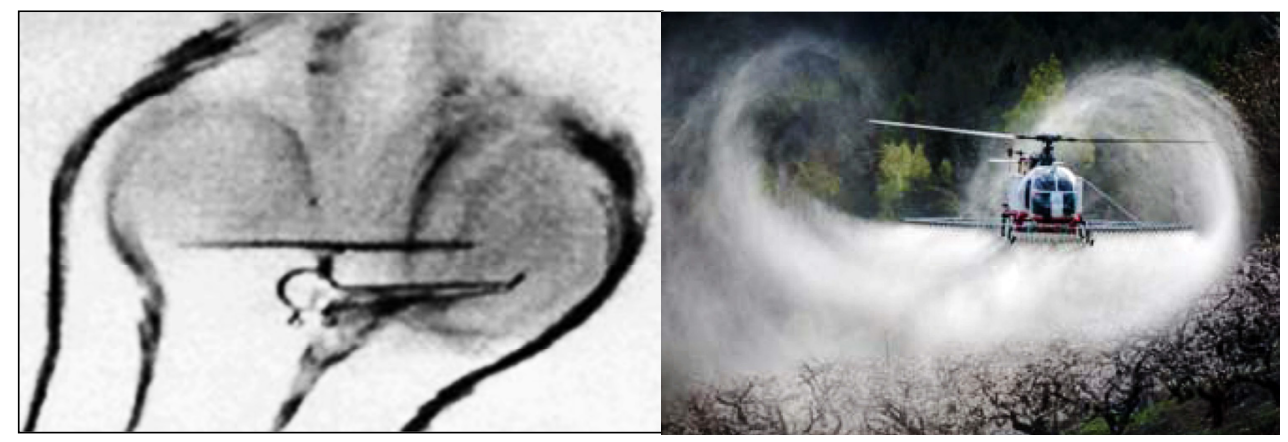

Fig. 1. Visualisation of vortex structures typical for the Vortex Ring State on a helicopter main rotor. Left: wind tunnel test (a smoke flow visualisation) [1]. Right: flight test [9].

Investigations of the VRS are very important from point of view of helicopter-flight safety. Therefore, many past and recent computational or experimental studies have been focused on this phenomenon [1, 3-10].

The presented study concerns computational investigations of the VRS. Compared to the state of the art, the research conducted is distinguishing by the new and original methodology of computer simulation of helicopter manoeuvres, based on the solution of Navier-Stokes equations but using some simplifications reducing an overall computational effort.

The presented research had two main goals. The first one was a development of methodology of computational simulation of a helicopter flight. The methodology should have been directed towards simulations of strongly unsteady manoeuvres, typical for an entrancing in or escaping from the VRS. The second goal of the research was a conduction of series of simulations of helicopter manoeuvres, focusing mainly on the identification of flight conditions leading to an occurrence of the VRS as well on the searching for optimal strategies to avoid or safely escape from the VRS.

\section{Methodology}

In the presented approach, the aerodynamic model of the rotorcraft flight is based on the solution of Unsteady Reynolds Averaged Navier-Stokes (URANS) equations describing the airflow in the region surrounding the flying helicopter. Simulation of unsteady flight or manoeuvres refers to the motion of the entire aircraft treated as a rigid body and is performed in relation to the time scale associated with this motion. The helicopter motion is modelled based on a system of equations describing the motion of a rigid body with six degrees of freedom. A simplified approach to a modelling of rotating blades of main and tail rotor has been applied. Aerodynamic forces generated by rotating blade systems (main rotors, tail rotors, propellers, etc.) and flow effects induced by these systems are determined using the approach named the Virtual Blade Model (VBM).

The general scheme of the developed methodology of rotorcraft flight simulation is presented in Fig. 2. The URANS equations are solved by the use of the ANSYS FLUENT software [2], being an industrial standard within the Computational Fluid Dynamic area. All aerodynamic forces and moments acting on modelled non-rotating solid walls, like a surface of fuselage, tail, tail boom, landing gear, etc., are calculated using standard routines of ASYS FLUENT. 
The rotorcraft flight simulation procedure is completely embedded in the solver ANSYS FLUENT that is supported by three User-Defined-Function (UDF) modules, being, in fact, the in-house software developed by the Authors. The UDF module "Virtual Blade Model" is responsible for modelling of flow effects caused by rotating rotor blades and for trimming of rotors or even complete helicopter. The UDF module "Flight Dynamics" is responsible for a solution 6-degree-of-freedom equations of aircraft motion. The UDF module "Kinematics" is responsible for modelling of rotorcraft motion and for realisation of helicopter-flight control.

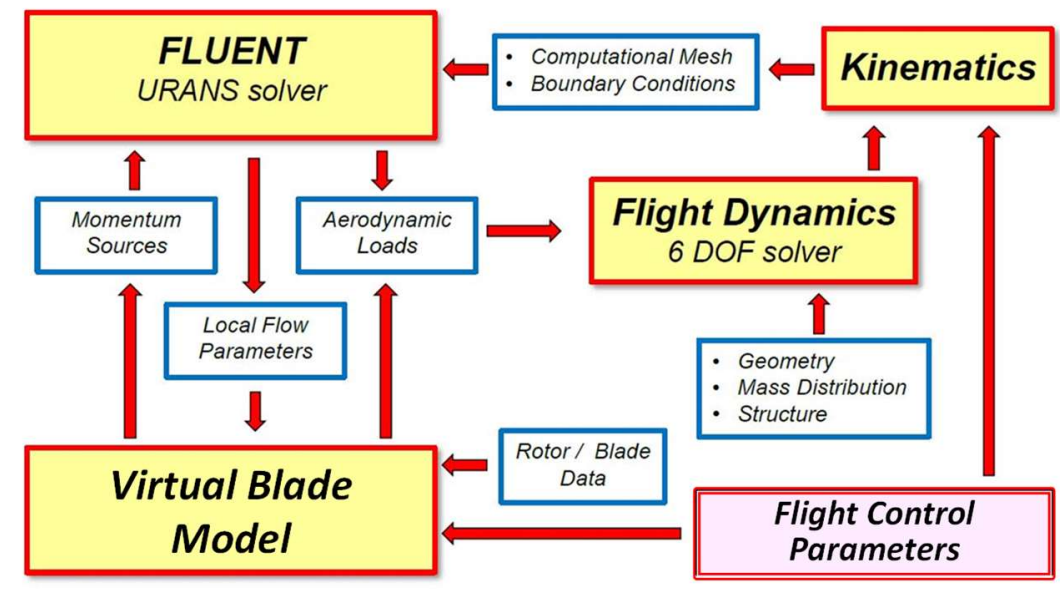

Fig. 2. General scheme of the developed methodology of rotorcraft-flight simulation.

In the simplified approach based on the Virtual-Blade-Model methodology, real rotors are replaced by volume discs influencing the flow field in a manner similar to real rotating blades. Time-averaged aerodynamic effects of rotating blades are modelled by means of artificial momentum sources placed inside the volume-disc zones localised in regions of rotation of real rotors. Such zones, replacing the real main and tail rotor are shown in Fig. 3. The intensities of momentum sources are evaluated based on the Blade Element Theory, associating local flow parameters (i.e. velocity vector, density, viscosity and sound speed) in rotor-disc zones with aerodynamic characteristics of airfoils - selected cross-sections of the blades. Databases of these characteristics (in general: lift and drag coefficients versus angle of attack tabularized for several sets of Mach and Reynolds numbers) should be prepared before starting the flight simulation. The original VBM code was significantly modified and expanded by the Authors of this paper. This especially concerned the development of methodology of controlling of helicopter flight.

Especially for the presented studies on the VRS, the original and advanced method of rotorcraft-flight control has been developed. In this approach, the helicopter flight is controlled by two systems.

The first one is an "autopilot", which means an automatic adjustment of the main-rotorblade collective and cyclic pitch as well as the tail-rotor-blade collective pitch, so as to obtain required values of:

- main rotor thrust,

- pitching, rolling and yawing moment acting on the helicopter.

The developed "autopilot" methodology utilises standard rotor-trimming procedures, implemented in the Virtual Blade Model methodology. This procedure consists in determination for a given rotor the collective and cyclic pitch of its blades so as to obtain required thrust and required pitching and rolling moments generated by this rotor [3]. 
The second system of flight control implemented in the presented methodology enables the user influencing manually the motion of the helicopter. In the presented approach this concerns the manual control of the following three parameters:

- pitch angle of the helicopter,

- bank angle of the helicopter,

- vertical (directed in the direction of gravity) component of helicopter acceleration.

These three parameters may be changed freely by the user during the flight simulation. If it is done, the "autopilot" system will trim the helicopter automatically, if possible.

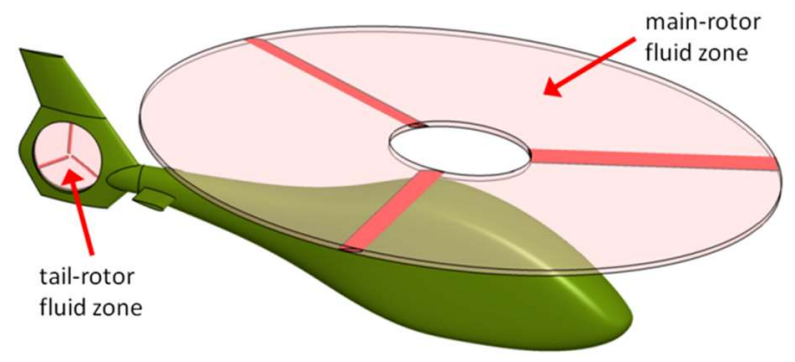

Fig. 3. Computational model of the helicopter.

Disc fluid zones as replacements of really rotating rotor blades.

\section{Helicopter flight simulations}

A series of flight simulations of the light helicopter equipped with a 3-blade main rotor was conducted. All simulations included the phases of an entrance in and escape from the VRS. The computational model of this helicopter is presented in Fig. 3. In general, this model includes all principal components of the helicopter: fuselage, tail boom, horizontal stabilizer, vertical tail, main rotor and tail rotor rotating inside the fenestron. The geometries of the main rotor and tail rotor were replaced by cylindrical fluid zones, in a manner typical for the VBM methodology, as shown in Fig. 3. All URANS simulations discussed below were conducted using the ANSYS FLUENT solver. The following flow model was assumed: three dimensional, unsteady, compressible, viscous, turbulent, with the Spalart-Allmaras turbulence model.

In all simulations, the flight of the helicopter, conducted in the motionless air, was modelled by the use of the Moving Reference Frame method, implemented in ANSYS FLUENT. In discussed below cases, the initial state of helicopter flight was a hover (out of ground effect) at altitude 500 meters above sea level, in ISA atmospheric conditions. This flight state was obtained through conducting a separate simulation aiming at the determination of initial conditions for the actual simulations.

In both discussed below simulations, the entrance in the VRS was conducted in the same way. Starting from the hover, the helicopter entered the horizontal flight through pitching its nose downward, thus generating component of main rotor thrust pulling the helicopter forward. To enter the VRS, the helicopter slowed down its horizontal flight, by pitching its nose upwards. Simultaneously, the vertical acceleration of the helicopter (one of "manual" flight controls utilised in the presented methodology) was set to $-2 \mathrm{~m} / \mathrm{s}^{2}$. As a result, the helicopter entered a steep descent. When the first symptoms of the VRS on the main rotor appeared, the helicopter started the escape from this dangerous flight state.

In single rotor helicopters, the classic way to escape from the VRS consists in slightly pitching nose down, and establishing forward flight. Such manoeuvre has been conducted in the first discussed below simulation. 
Another solution, applicable for both the single rotor and the tandem-rotor helicopters, is the Vuichard Recovery Technique [9-10], developed by Claude Vuichard, a Federal Office for Civil Aviation inspector in Switzerland. In this case, the escape from the VRS require to generate lateral force moving the helicopter sideward, in the direction of positive thrust of the tail rotor. To generate the side acceleration of the helicopter, both the main and tail rotor thrust is utilised. The Vuichard Recovery Technique has been applied in the second presented below simulation.

In both presented helicopter-flight simulations, after successful escape from the VRS, the helicopter gradually recovered a flight altitude $(500 \mathrm{~m})$ and eventually passed to a horizontal flight.

Within the first presented simulation, the classic escape from the VRS was conducted. The simulation consisted in the entrance in VRS during a steep descent then the escape from VRS through a forward-accelerated flight. Fig. 4 presents the three-dimensional view of the flight trajectory and its and its two-dimensional projections on main planes of the coordinate system. The position of helicopter at the beginning of the escape from VRS is marked on the trajectory by a pink circle. The upper graph in Fig. 5 shows user-defined time-varying helicopter flight control parameters. Presented runs of helicopter vertical acceleration and its pitch and bank angles, were adjusted by the user so as to obtain required flight trajectory shown in Fig. 4 and required flight velocities presented in the lower graph in Fig. 5.

Fig. 6 presents visualisations of flow around the helicopter, captured in selected phases of escape from VRS, i.e.: beginning of the escape, its essential phase and the final phase, when the vortex ring is decaying. In the upper part of Fig. 6, the flow velocity field is visualised in a cross-section through the centre of the main rotor. In these visualisations the vortex cores are marked by violet circles. In the final phase of the escape, the vortex structures are hardly visible. In the lower part of Fig. 6, the streamlines flowing from the main and tail rotor discs are visualised. Additionally, the blue broken line represents the helicopter trajectory. One can notice that in the initial and in the essential stage of the escape, the vortex ring is well visible. However, at the final stage of the escape, it is blown off of the main rotor disk in a direction opposite to a helicopter flight direction.

Within the second simulation, the Vuichard Recovery Technique was applied as a means to escape from the VRS. The simulation consisted in the entrance in VRS during a steep descent then the escape from VRS through a sideward-accelerated flight. Fig. 7 presents the three-dimensional view of the flight trajectory together with marked the position of helicopter at the beginning of the escape from VRS. Up to this point, the helicopter trajectory is the same as in the previous case. However, when escaping from VRS, the trajectory goes sideways as per the Vuichard's technique. For the helicopter to move along such a trajectory, flight control parameters (i.e. helicopter vertical acceleration and its pitch and bank angles) were specially adjusted, as shown in the upper part of Fig. 8. In the lower part of Fig. 8, the flight velocity and descent rate are presented as functions of time.

Fig. 9 presents visualisations of flow around the helicopter, captured in selected phases of sideward escape from VRS. Compared to Fig. 6, in the discussed case, the vortex ring is blown off of the main rotor disk in a lateral direction relative to the helicopter longitudinal axis. 


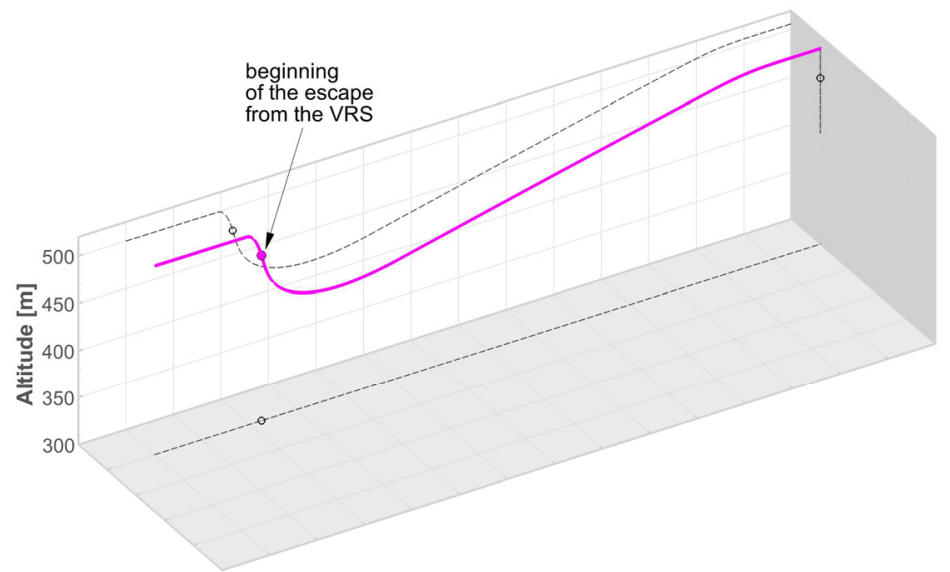

Fig. 4. Flight trajectory in the case of the escape from VRS through a forward-accelerated flight.
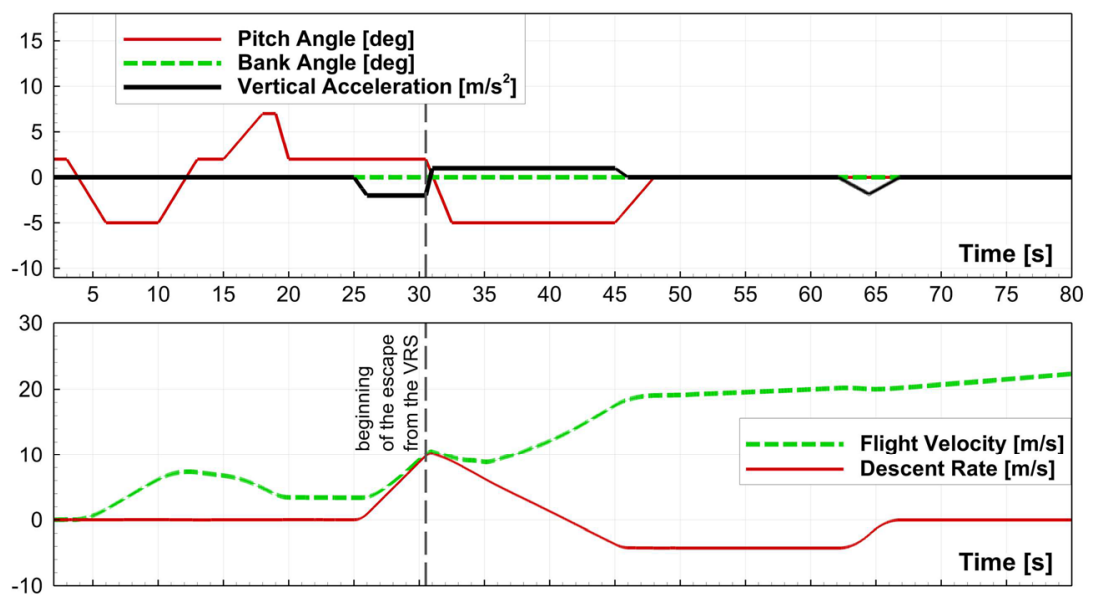

Fig. 5. Simulation of the escape from VRS through a forward-accelerated flight.

Upper: flight control parameters vs. time. Lower: flight velocity and descent rate vs. time.

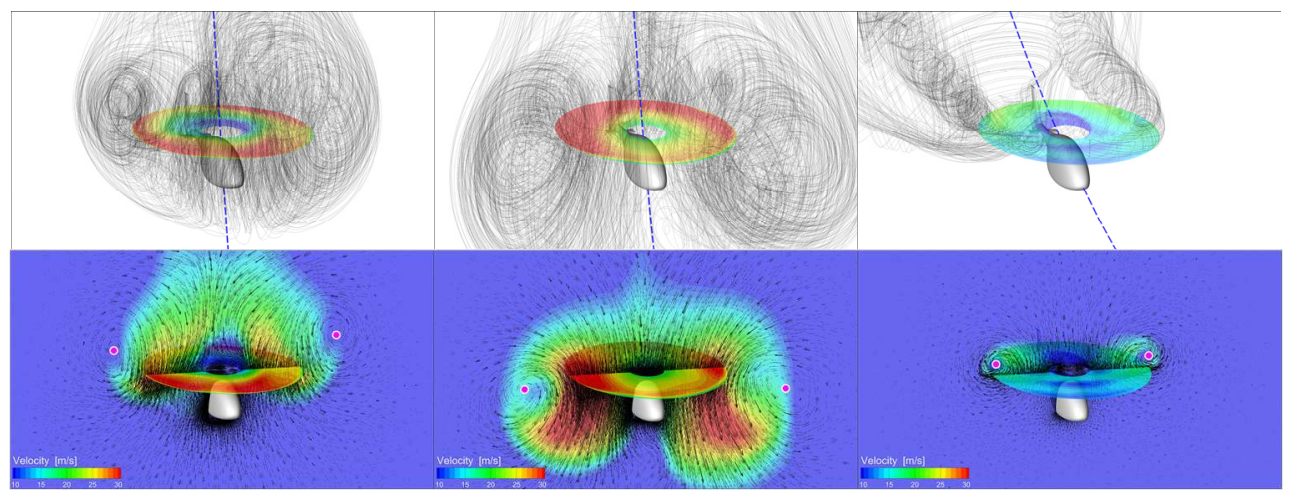

Fig. 6. Simulation of the escape from VRS through a forward-accelerated flight.

Upper row: streamlines flowing from the main and tail rotor discs.

Lower row: magnitude and direction of the flow velocity vector in a flow field cross-section.

Left: beginning of the escape from VRS. Middle: essential phase of the escape.

Right: decay of the vortex ring in the final phase of the escape. 


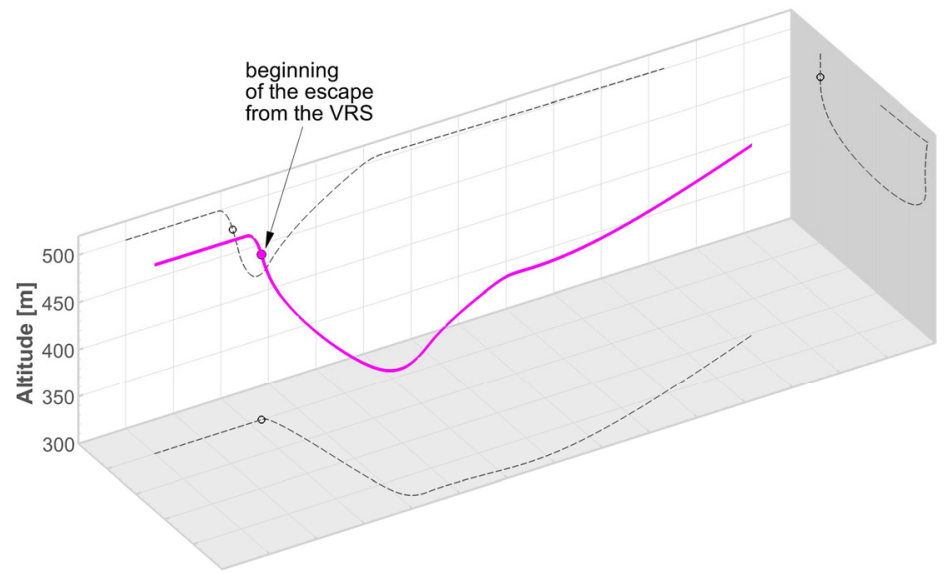

Fig. 7. Flight trajectory in the case of the escape from VRS through a sideward-accelerated flight.
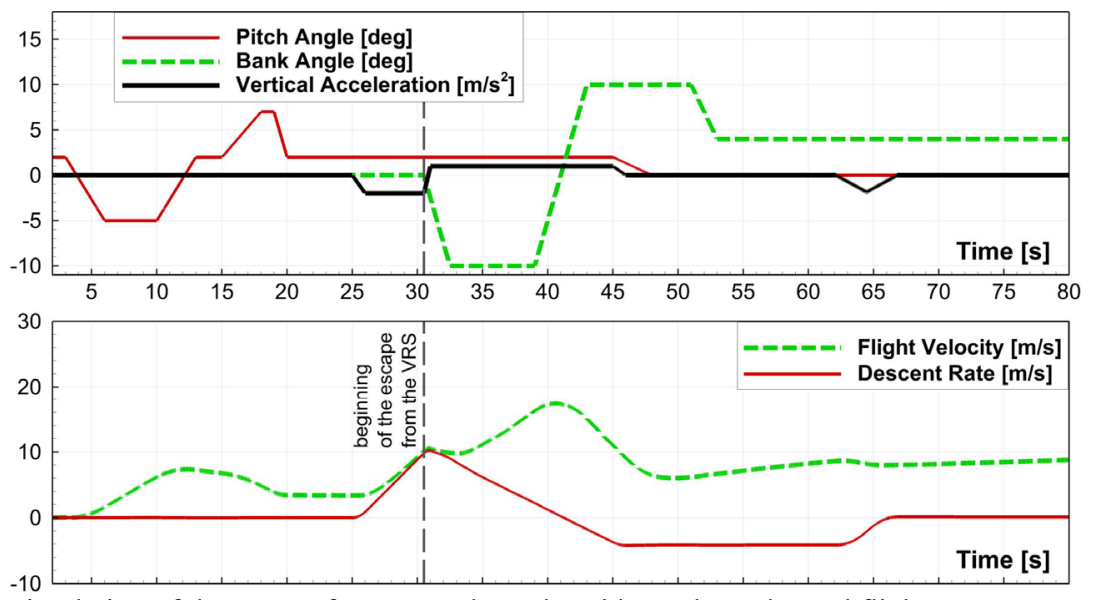

Fig. 8. Simulation of the escape from VRS through a sideward-accelerated flight.

Upper: flight control parameters vs. time. Lower: flight velocity and descent rate vs. time.

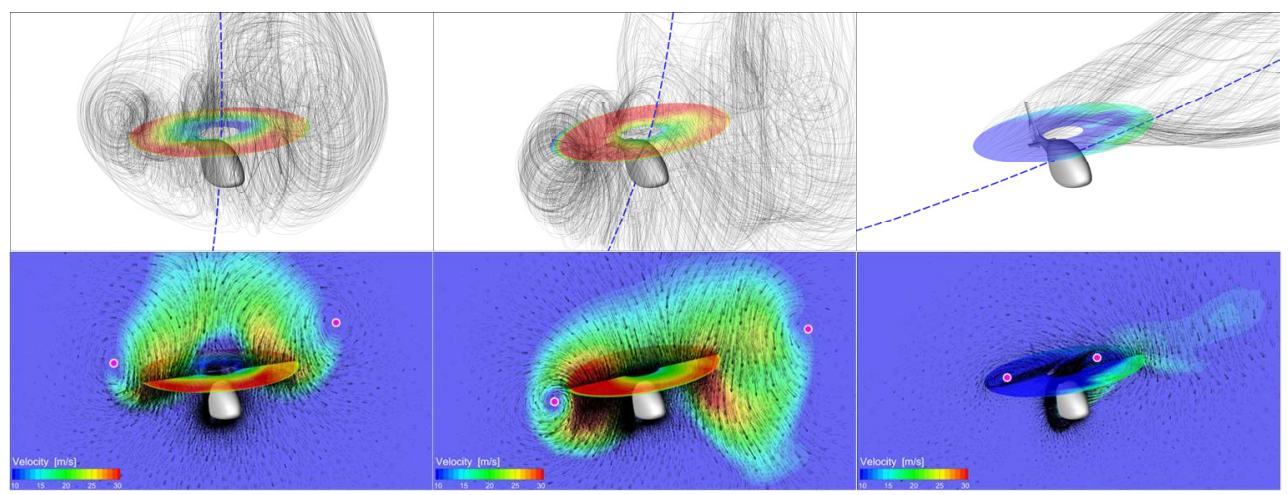

Fig. 9. Simulation of the escape from VRS through a sideward-accelerated flight.

Upper row: streamlines flowing from the main and tail rotor discs.

Lower row: magnitude and direction of the flow velocity vector in a flow field cross-section.

Left: beginning of the escape from VRS. Middle: essential phase of the escape.

Right: decay of the vortex ring in the final phase of the escape. 


\section{Conclusions}

The developed and implemented methodology of computational simulation of strongly unsteady manoeuvres of a helicopter, seems to be a valuable and reliable tool, in particular useful when investigating the Vortex Ring State - a very dangerous phenomenon that may occur during a helicopter flight.

The presented results of conducted simulations of helicopter manoeuvres, qualitatively comply with both the results of experimental studies and flight tests, cited in literature. The future continuation of the presented study will primarily include quantitative validation of the developed methodology, in respect to well-documented flight tests of real helicopters.

Reliable computer simulations performed using the developed methodology can significantly contribute to increased helicopter flight safety.

\section{References}

1. J. Drees, W.P. Hendal, Airflow patterns in the neighbourhood of helicopter rotors, Aircraft engineering, Volume 23 / 266 (1951)

2. ANSYS, Inc., ANSYS FLUENT User's Guide. Release 18.2. Available from: http://www.ansys.com

3. D. Peters, D. Barwey, A general theory of rotorcraft trim, Mathematical Problems in Engineering, Volume 2, pp. 1-34 (1996).

4. W. Johnson, Model for vortex ring state influence on rotorcraft flight dynamics, NASA, TP-2005-213477, California (2005)

5. B. N. Juriew, Aerodynamics of propellers and helicopters, WMON, Moscow (1956)

6. J. Pegg, An investigation of the helicopter height-velocity diagram showing effects of density altitude and gross weight, NASA, TN D-4536, (1968)

7. J. Seddon, Basic helicopter aerodynamics, BSP Professional Books, Oxford (1990)

8. W. Stewart, Helicopter Behaviour in the Vortex-Ring Conditions, Aeronautical Research Council Reports and Memoranda, Her Majesty's Stationery Office, London (1959)

9. The Vuichard Recovery, International Helicopter Safety Foundation, Airmanship Bulletin, Available from: http://www.IHST.org

10. T. Tucker, Flying Through the Vortex, Rotor \& Wing. Aviation Today (2016)

11. Robinson R22/R44 Flight Training Guide, R22 Maneuver guide, Settling-WithPower/Vortex Ring State, (2013) 